\title{
BMP-2 Promotes Bone Formation in Bone Defects in Which Bone Remodeling Is Suppressed by Long- Term and High-Dose Zoledronic Acid
}

\section{Young Jae Moon}

Jeonbuk National University Medical School

\section{Seongyup Jeong}

Jeonbuk National University Medical School and Hospital

Kwang-Bok Lee ( $D$ osdr2815@naver.com)

Jeonbuk National University Medical School

\section{Research Article}

Keywords: Bisphosphate, zoledronic acid, bone morphogenetic protein, bone defect, bone remodeling, atypical fracture

Posted Date: September 1st, 2021

DOI: https://doi.org/10.21203/rs.3.rs-684348/v2

License: (9) This work is licensed under a Creative Commons Attribution 4.0 International License. Read Full License 


\section{Abstract}

Background: The use of long-term and high-dose bisphosphate is associated with severely suppressed bone turnover and the delayed union of fractures. However, therapeutic methods to overcome the negative effects of bisphosphonate use are lacking. Bone morphogenetic proteins (BMPs) are powerful osteoinductive proteins. We hypothesized that BMPs had similar effects as autografts in patients with decreased bone healing potential due to long-term bisphosphonate treatment. The purpose of this study was to compare BMPs with demineralized freeze-dried bone grafts and autografts in a rat femoral bone defect model with long-term and high-dose bisphosphonate treatment.

Methods: Forty rats were divided into the following four groups depending upon the materials implanted into the femoral defect after ten weeks of bisphosphonate (zoledronic acid) injections: Group I: absorbable collagen sponge (control); group II: demineralized freeze-dried bone graft; group III: autogenous bone graft; and group IV: rhBMP-2 with an absorbable collagen sponge. Radiographic union, micro computed tomography (CT) analysis, manual palpation, and histologic analysis were evaluated.

Results: The radiographic union rate, manual union rate, and micro-CT bone volume in groups III and IV were significantly higher than those in groups I and II. Groups III and IV showed similar results to each other. Although the amount of immature bone in the BMP-treated group was large, the effect was similar to that of autografts in the bone defect model in which bone turnover was severely reduced by bisphosphonate treatment.

Conclusion: BMP might be a good substitute for autografts in patients with decreased bone healing potential due to long-term bisphosphonate treatment.

\section{Background}

Bisphosphonates are widely used to treat osteoporosis because they are believed to prevent bone loss by inhibiting osteoclast activity [1]. These drugs increase bone strength and reduce the risk of fracture in patients with osteoporosis, Paget's disease, and metastatic bone disease [2-4]. However, because osteoclasts have a major role in the fracture repair and remodeling of bone graft sites, long-term and high-dose bisphosphonate use impaired callus remodeling and the healing of bone defect sites in animal models [5-7]. Clinically, the long-term use of bisphosphonates in osteoporotic patients or high doses of bisphosphonates used for the treatment of osteogenesis imperfecta and metastatic bone tumors leads to atypical femoral fractures [8-10]. In addition, case series have shown that the long-term use of bisphosphonates increased the occurrence of nonunions after intramedullary nailing treatment for atypical femur fractures $[11,12]$. However, there are still limited treatment choices after an atypical fracture due to long-term and high-dose bisphosphonate treatment.

Bone morphogenetic proteins (BMPs) are a subgroup of the transforming growth factor- $\beta$ (TGF- $\beta$ ) superfamily [13-15]. Many studies have reported that BMPs can accelerate bone regeneration and fracture healing $[14,16]$. Furthermore, BMPs are as effective as autogenous bone grafts for fracture 
healing [17]. The potential benefits of using BMPs include decreased donor-site morbidity associated with harvesting the autograft and reduced costs associated with treatment for delayed unions and nonunions [18].

We hypothesized that BMPs had a similar effect as autografts on the union rate of fractures in patients with decreased bone healing potential due to long-term and high-dose bisphosphonate treatment. Therefore, the objective of this study was to compare BMPs with demineralized freeze-dried bone (DFDB) and autografts in a rat femoral defect model with long-term and high-dose bisphosphonate treatment.

\section{Methods}

\section{Study design}

This study was approved by the Institutional Animal Care and Use Committee (IACUC) of the Jeonbuk National University Laboratory Animal Center, Jeonbuk National University, Korea. All animals were cared for in accordance with the regulations of the IACUC under the supervision of licensed veterinarians. The study was conducted according to the ARRIVE guidelines for the reporting of animal experiments. The surgical procedures and assessment of the unions were performed according to the methods of Liao et al [13].

Forty rats were divided into the following four groups, and ten rats were assigned to each group:

Group I: absorbable collagen sponge (ACS) (Gelfoam; Mascia Brunelli Spa, Milano, Italy) alone group; group II: DFDB graft group-cancellous bone chips (Community Tissue Services, Dayton, OH, USA); group III: autogenous bone graft group (iliac cortico-cancellous bone); group IV: rhBMP-2 implant group (Daewoong CG Bio Pharmaceutical Co. Ltd., Seoul, Korea) with ACS.

Pathogen-free 12-week-old female Lewis rats $(230$ - $290 \mathrm{~g})$ were purchased from Orient Bio (Seoul, Korea). They were housed in a laminar flow cabinet with a $12 \mathrm{~h}$ light/dark cycle and maintained on standard laboratory chow ad libitum. Each rat was given an intraperitoneal injection of $0.1 \mathrm{mg} / \mathrm{kg}$ zoledronic acid (Zometa) once a week for 10 weeks. After that, femoral bone defect surgery was performed on all rats. All rats underwent radiographic studies every two weeks after the surgical procedure. Sixteen weeks after the operation, the bone volume in the defect site of the femur was assessed by micro-computed tomography. All animals were euthanized at 16 weeks after surgery and their right femurs were harvested. Union rate determination, manual palpation, and histologic studies were performed for the defect site of the femur.

\section{Surgical Procedures}

All rats were anesthetized using Zoletil (Virbac, France) intraperitoneally $(7 \mathrm{mg} / \mathrm{kg})$. The right hind limb was shaved and disinfected with alcohol and povidone-iodine. A longitudinal 3-cm skin incision was made in the lateral aspect of the femur and the entire length of the right femur was exposed. The periosteum was stripped from the shaft. A 5-mm mid-diaphyseal full-thickness defect was created using 
a bone saw. A polyethylene plate $(23 \mathrm{~mm} \times 4 \mathrm{~mm} \times 4 \mathrm{~mm})$ was secured with four $0.99-\mathrm{mm}$ Kirschner wires and two 0.53-mm steel cerclage wires on the lateral side of the femur, as previously described [13]. Each testing material was inserted into the defect in groups II, III, and IV. ACS only was inserted into group I. Iliac cortico-cancellous bone was used for the autograft group. We performed an oblique 2-cm skin incision over the posterior superior iliac spine (PSIS) to retract the soft tissue and expose the PSIS. After cortical osteotomy, cortico-cancellous bone grafts were taken using a small-size curette. Autobone and DFDB were grafted in $0.5 \mathrm{cc}$ amounts. For accurate grafting, we measured $0.5 \mathrm{cc}$ using a 2-cc syringe. rhBMP was implanted at $10 \mu \mathrm{g} / 0.5 \mathrm{cc}$. In the femoral defect model, $10 \mu \mathrm{g}$ BMP was most effective in inducing bone formation $[19,20]$.

\section{Radiographic Evaluation}

All animals were anesthetized and radiographs were taken every two weeks. The rats were imaged with a high-resolution digital mammographic imager (Mammomat Novation, Siemens AG Medical Solutions, Erlangen, Germany). All images were obtained with exposure settings of $34 \mathrm{kVp}$ and $110 \mathrm{~mA}$ at a magnification of $1.5 \times$. Radiographic union was assessed by bony bridge formation of the cortex. $A$ complete union was defined as the bony bridge formation of both cortexes in radiography while a partial union was defined as the bony bridge formation of one cortex. Nonunion was defined as no bony bridge formation.

\section{Micro-CT Analysis}

Sixteen weeks after surgery, all rats underwent micro-CT scanning (NFR Polaris-G90, NanoFocusRay Co., LTF., Jeonju, Korea) to evaluate bone volume and healing of the defect site. The scanner was set at an Xray voltage of $70 \mathrm{kVp}$ with an X-ray current of $100 \mu \mathrm{A}$. The scans were completed over $360^{\circ}$ of rotation of the $\mathrm{X}$-ray tube. All micro-CT image data were acquired using live, free-breathing rats anesthetized by intraperitoneal Zoletil $\$ injections. We measured the bone volumes around the defect site using a microCT scanner (NFR Polaris-G90). A cylindrically shaped region of interest (ROI) was established with the middle of the bone defect site as the center, which had a diameter of $14.25 \mathrm{~mm}$ and a height of $6.65 \mathrm{~m}$. The bone volume (BV), tissue volume (TV), and bone volume (BV/TV) percentage were measured in the ROI.

\section{Manual Palpation and Manipulation}

Manual palpation is a sensitive and specific method of assessing bone fusion [13,21-23]. Manual palpation with varus/valgus and anterior/posterior angulated force was performed for all harvested specimens with a particular focus at the defect region. All specimens were manipulated with a force high enough to evaluate the gross motion. When no motion was present, it was considered a complete union, as previously described [13].

\section{Histology Analysis}


We performed bony biopsies of the resected femurs to evaluate the bone healing potential. The resected femurs and jaws were fixed in 10\% neutral buffered formalin and decalcified in $10 \%$ ethylenediaminetetraacetic acid (EDTA) for 10 days or in rapid decalcifying solution (Calci-Clear Rapid, National Diagnostics, Atlanta, GA, USA) for 12 to $24 \mathrm{~h}$. To evaluate the histologic changes, paraffinembedded tissue sections were stained with hematoxylin (Sigma-Aldrich, St. Louis, MO, USA).

\section{Statistical Analysis}

Statistical analysis was carried out with Statistical Package for Social Sciences software (SPSS Inc., Seoul, Korea). The union rates detected by radiological evaluation (union rate), the manual test, and the bone volume in micro-computed tomography were compared using one-way analysis of variance (ANOVA) with a post-hoc test (Scheffe test). P-values of less than 0.05 were considered statistically significant.

\section{Results}

All rats tolerated the surgical procedures. There were no serious complications after the surgeries.

\section{The effect of long-term treatment with bisphosphonate}

Long-term and high-dose bisphosphonate injections for osteoporosis treatment are associated with complications such as osteonecrosis characterized by empty lacunae in the deeper layer of bone and chronic inflammation in the bone marrow [24]. To confirm whether long-term and high-dose bisphosphonate worked in this model, we histologically observed the distal femur of rats treated with long-term and high-dose zoledronate. As expected, empty lacunae were observed in more than $50 \%$ of the osteocyte lacunae in the distal femur and the infiltration of chronic inflammatory cells was observed in the bone marrow cavity (Fig. 1). Furthermore, metaphyseal bands, which are called zebra lines, were observed in the proximal tibia (Fig. 2; yellow arrows). Zebra lines were observed near the ends of the long bones during cyclic bisphosphonate therapy for osteogenesis imperfect [25].

\section{Radiographic Evaluation}

The results of the radiographic evaluation are summarized in Table 1. Sixteen weeks after the surgical procedure, radiographic complete unions were achieved in six rats in group III, six rats in group IV, two rats in group II, and none in group I. Partial unions were achieved in one, two, three, and two rats of groups I, II, III, and IV, respectively (Fig. 2). The partial and complete union rates of groups III and IV were significantly higher than those of groups I and II. There were no significant differences between groups III and IV and between groups I and II.

\section{Manual Palpation and Manipulation}


The results of the manual palpation for union (Table 2) were similar to the radiographic results. Complete unions were achieved in six rats in group III, six rats in group IV, two rats in group II, and none in group I. In the manual test, the union rates of the rats in groups III and IV were significantly $(p<0.05)$ higher than those of groups I and II.

\section{Micro-CT Analysis - Bone Volume}

The three-dimensional (3D) reconstruction images are shown in Figure 3. The results of the micro-CT analysis for bone volume are shown in Table 3 . The mean bone volumes in groups I, II, III, and IV were $42.8 \pm 19.8 \mathrm{~mm}^{3}, 72.93 \pm 15.6 \mathrm{~mm}^{3}, 168.78 \pm 20.1 \mathrm{~mm}^{3}$, and $193.94 \pm 61.5 \mathrm{~mm}^{3}$, respectively. The mean bone volumes of groups III and IV were significantly $(p<0.05)$ larger than those of groups I and II. However, there was no significant $(p>0.05)$ difference in bone volume between groups III and IV. The mean bone volume per tissue volume (BV/TV) percentage in the II II, III, and IV groups was $15.79 \pm 5.6 \%$, $36.44 \pm 2.5 \%, 64.3 \pm 1.4 \%$, and $56.59 \pm 9.8 \%$, respectively. The mean BV/TV of groups III and IV was significantly higher than that of groups I and II. In contrast to the bone volume pattern, the mean BV/TV value in group III was increased compared to that in group IV, but the increase was not significant. These results suggested that the autobone grafts had a small amount of callus, but the remodeling potential was faster than that of BMP.

\section{Histological Analysis}

In the histological analysis of the resected femurs, the bone defect sites in the absorbable collagen sponge alone group were filled with fibrous tissue without bone formation. In the DFDB graft group, the defect site was filled with fragmented lamellar bone. Lamellar bone in the defect sites of the bone autografts was more abundant and thicker with relatively less fibrous tissue compared to that of the DFDB graft group. However, the junction between the cortical bone and the newly formed bone in the autografts was partially discontinuous. In contrast, the defect site in the BMP-treated group was filled with newly formed bone and the junction between the cortical bone and new bone was continuous (Fig. $4)$.

\section{Discussion}

Our findings demonstrated the potential of rhBMP for bone formation even in bone defects in which bone remodeling was severely reduced by long-term and high-dose zoledronic acid treatment. Although the BMP-induced bone formation seemed immature compared to that of the autogenous bone grafts, the manual tests and histological findings showed that BMP had a similar effect on the bony union as autogenous bone grafts in severely suppressed bone turnover conditions. Based on these results, BMP might be used as an effective substitute for autografts in patients with long-term and high bisphosphonate treatment.

The novel finding of this study was the effect of BMP on bone defects in rats with severely suppressed bone turnover. Recent studies on the association of bisphosphonate and BMP reported a positive effect 
of bisphosphonate in the inhibition of osteoclast activity, compared between a group treated with BMP alone and a group treated with bisphosphonates and BMP simultaneously after the fracture [21, 26, 27]. These studies focused on the temporary suppression of osteoclast activity after a fracture. However, clinically, long-term and high-dose bisphosphonates use inhibited bone turnover, accumulated microfractures, reduced energy absorption, increased the fragility of osteoporotic bones, and inhibited bone remodeling through osteocyte death, eventually leading to atypical fractures $[6,9,28]$. It also promoted nonunion after the treatment of these fractures $[11,12]$. The present study focused on these side effects of bisphosphonates and confirmed the role of BMP.

For surgeries that need bone grafts such as cases of nonunion or osteotomy, most surgeons may perform autogenous bone grafts. However, the osteogenic effect and the amount of the autogenous bone used are lower in older patients than in younger patients [29]. Moreover, in older patients with long-term bisphosphonate treatment, the union rate of osteotomies or nonunions might be low even if an autogenous bone graft is undertaken [30]. Therefore, alternatives to autogenous bone are needed. Our results suggested that BMP might be a suitable substitute for autogenous bone in patients with poor bone quality. Supporting our results, the efficacy of BMP as an enhancer of bone repair has already been confirmed in several studies of segmental bone defects or fractures [31,32]. Multiple clinical trials have shown that BMP and autogenous bone grafts had similar fracture healing rates, with reduced blood loss and shortened surgery time, without donor site morbidities [16, 33].

\section{Limitations}

This study had several limitations. First, the amount injected or the period of bisphosphonate injections might not have been sufficient to decrease the bone healing potential of the femur in this study. In the femoral defect model in this study, whether the bone healing potential was actually decreased in the femur was unclear. To resolve this limitation, we performed intraperitoneal injections of $0.1 \mathrm{mg} / \mathrm{kg}$ zoledronic acid once a week for 10 weeks according to a previous study [34]. Through bone biopsies of the femurs, we identified empty cortical lacunae and assumed that the bone healing potential in the femur was decreased. Second, the rats used in this study were 12 weeks old. Because most humans treated with long-term bisphosphonates are older patients with osteoporosis, the bone healing process in the autografts of young rats used in this study was much more effective than that in older humans. Nevertheless, the complete union rates and bone volume between the autograft group and the BMP group were similar to each other. Therefore, BMP might be more effective than autografts in older patients. Third, we did not include a group of rats that did not receive zoledronic acid and groups with different concentrations of BMP. Because it had already been reported that BMP facilitated bone healing and $10 \mathrm{ug}$ of BMP was the optimal dose in the femoral bone defect model, we did not include a zoledronic acid nontreated group and used $10 \mathrm{ug}$ of BMP in the experiments $[19,20]$. Fourth, we did not perform biomechanical testing that could quantify the degree of bony union. Instead, we evaluated bony union by the manual palpation method [13, 21-23]. 


\section{Conclusions}

We demonstrated that BMP and autografts had similar effects on the union rate and bone volume of rats with long-term and high-dose bisphosphonate treatment. This study suggested that BMP would be useful in promoting the repair of atypical fractures and the nonunion of atypical fractures.

\section{Abbreviations}

BMP: Bone morphogenetic proteins

CT: Computed tomography

DFDB: Demineralized freeze-dried bone

ACS: Absorbable collagen sponge

BV: Bone volume

TV: Tissue volume

\section{Declarations}

\section{Acknowledgment}

This study was supported by a grant from the CNUH-BRI (Biomedical Research Institute of Chonbuk National University Hospital, CNUH-BRI-2012-02-005).

\section{Author contributions}

K.B.L. designed the research; Y.J.M. and S.J performed the research; Y.J.M. analyzed the data; K.B.L. provided financial support; Y.J.M and K.B.L wrote the paper.

\section{Availability of data and materials}

The datasets used and/or analyzed during the current study are available from the corresponding author on reasonable request.

\section{Ethic approval and consent to participate}

The experimental protocol was established according to the ethical guidelines and was approved by the Institutional Animal Care and Use Committee (IACUC) of the Jeonbuk National University Laboratory Animal Center, Jeonbuk National University, Korea, and followed the ARRIVE guidelines and Institutional Guidelines for the care and use of animals. 
Not applicable

\section{Competing interests}

The authors declare that they have no competing interests.

\section{References}

1. Luckman SP, Hughes DE, Coxon FP, et al. Nitrogen-containing bisphosphonates inhibit the mevalonate pathway and prevent post-translational prenylation of GTP-binding proteins, including Ras. J Bone Miner. Res. 1983;13(4):581-9. https://doi.org/10.1359/jbmr.1998.13.4.581.

2. Black DM, Delmas PD, Eastell R, et al. Once-yearly zoledronic acid for treatment of postmenopausal osteoporosis. N Engl J Med. 2007;356(18):1809-22. https://doi.org/10.1056/NEJMoa067312.

3. Hosking D, Lyles K, Brown JP, et al. Long-term control of bone turnover in Paget's disease with zoledronic acid and risedronate. J Bone Miner Res. 2007;22(1):142-8. https://doi.org/10.1359/jbmr.061001.

4. Ross JR, Saunders Y, Edmonds PM, et al. Systematic review of role of bisphosphonates on skeletal morbidity in metastatic cancer. BMJ. 2003;327(7413):469. https://doi.org/10.1136/bmj.327.7413.469.

5. Hauser M, Siegrist M, Denzer A, et al. Bisphosphonates reduce biomaterial turnover in healing of critical-size rat femoral defects. J Orthop Surg (Hong Kong). 2018;26(3):2309499018802487. https://doi.org/10.1177/2309499018802487.

6. Kates SL. Ackert-Bicknell CL. How do bisphosphonates affect fracture healing? Injury. 2016;47 SuppI 1(0 1):S65-8. https://doi.org/10.1016/S0020-1383(16)30015-8.

7. Pozzi S, Vallet S, Mukherjee S, et al. High-Dose Zoledronic Acid Impacts Bone Remodeling with Effects on Osteoblastic Lineage and Bone Mechanical Properties. Clin Cancer Res. 2009;15(18):5829-39. https://doi.org/10.1158/1078-0432.CCR-09-0426.

8. Puhaindran ME, Farooki A, Steensma MR, et al. Atypical subtrochanteric femoral fractures in patients with skeletal malignant involvement treated with intravenous bisphosphonates. J Bone Joint Surg Am. 2011;93(13):1235-42. https://doi.org/10.2106/JBJS.J.01199.

9. Shane E, Burr D, Abrahamsen B, et al. Atypical subtrochanteric and diaphyseal femoral fractures: second report of a task force of the American Society for Bone and Mineral Research. J Bone Miner Res. 2014;29(1):1-23. https://doi.org/10.1002/jbmr.1998.

10. Vasanwala RF, Sanghrajka A, Bishop NJ, et al. Recurrent Proximal Femur Fractures in a Teenager With Osteogenesis Imperfecta on Continuous Bisphosphonate Therapy: Are We Overtreating? J Bone Miner Res. 2016;31(7):1449-54. https://doi.org/10.1002/jbmr.2805.

11. Grady MK, Watson JT, Cannada LK. Treatment of femoral fracture nonunion after long-term bisphosphonate use. Orthopedics. 2012;35(6):e991-5. https://doi.org/10.3928/01477447-2012052551. 
12. Nishino T, Hyodo K, Matsumoto $Y$, et al. Surgical results of atypical femoral fractures in long-term bisphosphonate and glucocorticoid users - Relationship between fracture reduction and bone union. J Orthop. 2019;19:143-9. https://doi.org/10.1016/j.jor.2019.11.044.

13. Liao JC, Tzeng ST, Keorochana G, et al. Enhancement of recombinant human BMP-7 bone formation with bmp binding peptide in a rodent femoral defect model. J Orthop Res. 2011;29(5):753-9. https://doi.org/10.1002/jor.21252.

14. Lissenberg-Thunnissen SN, de Gorter DJ, Sier CF, et al. Use and efficacy of bone morphogenetic proteins in fracture healing. Int Orthop. 2011;35(9):1271-80. https://doi.org/10.1007/s00264-0111301-z.

15. van Baardewijk LJ, van der Ende J, Lissenberg-Thunnissen S, et al. Circulating bone morphogenetic protein levels and delayed fracture healing. Int Orthop. 2013;37(3):523-7. https://doi.org/10.1007/s00264-012-1750-z.

16. Ghodadra N, Singh K. Recombinant human bone morphogenetic protein-2 in the treatment of bone fractures. Biologics. 2008;2(3):345-54. https://doi.org/10.2147/btt.s3394.

17. Jones AL, Bucholz RW, Bosse MJ, et al. Recombinant human BMP-2 and allograft compared with autogenous bone graft for reconstruction of diaphyseal tibial fractures with cortical defects. A randomized, controlled trial. J Bone Joint Surg Am. 2006;88(7):1431-41. https://doi.org/10.2106/JBJS.E.00381.

18. Axelrad TW, Steen B, Lowenberg DW, et al. Heterotopic ossification after the use of commercially available recombinant human bone morphogenetic proteins in four patients. J Bone Joint Surg Br. 2008;90(12):1617-22. https://doi.org/10.1302/0301-620X.90B12.20975.

19. Angle SR, Sena K, Sumner DR, et al. Healing of rat femoral segmental defect with bone morphogenetic protein-2: a dose response study. J Musculoskelet Neuronal Interact. 2012;12(1):2837.

20. Morishita $Y$, Naito M, Miyazaki M, et al. Enhanced effects of BMP-binding peptide combined with recombinant human BMP-2 on the healing of a rodent segmental femoral defect. J Orthop Res. 2010;28(2):258-64. https://doi.org/10.1002/jor.20970.

21. Doi Y, Miyazaki M, Yoshiiwa T, et al. Manipulation of the anabolic and catabolic responses with BMP2 and zoledronic acid in a rat femoral fracture model. Bone. 2011;49(4):777-82. https://doi.org/10.1016/j.bone.2011.07.005.

22. Kanezaki S, Miyazaki M, Ishihara T, et al. Enhancement of the effects of intermittent parathyroid hormone $(1-34)$ by bone morphogenetic protein in a rat femoral open fracture model. J Orthop Surg Res. 2019;14(1):403. https://doi.org/10.1186/s13018-019-1470-9.

23. Miyazaki M, Toyoda M, Yoshiiwa T, et al. Enhancement of the effects of exfoliated carbon nanofibers by bone morphogenetic protein in a rat femoral fracture model. J Orthop Res. 2015;33(2):185-92. https://doi.org/10.1002/jor.22751.

24. Sharma D, Ivanovski S, Slevin M, et al. Bisphosphonate-related osteonecrosis of jaw (BRONJ): diagnostic criteria and possible pathogenic mechanisms of an unexpected anti-angiogenic side 
effect. Vasc Cell. 2013;5(1):1. https://doi.org/10.1186/2045-824X-5-1.

25. Muderis MA, Azzopardi T, Cundy P. Zebra lines of pamidronate therapy in children. J Bone Joint Surg Am. 2007;89(7):1511-6. https://doi.org/10.2106/JBJS.F.00726.

26. Bosemark $P$, Isaksson $H$, Tagil M. Influence of systemic bisphosphonate treatment on mechanical properties of BMP-induced calluses in a rat fracture model: comparison of three-point bending and twisting test J Orthop Res. 2014;32(5):721-6. https://doi.org/10.1002/jor.22599.

27. Mathavan $\mathrm{N}$, Bosemark $\mathrm{P}$, Isaksson $\mathrm{H}$, et al. Investigating the synergistic efficacy of BMP-7 and zoledronate on bone allografts using an open rat osteotomy model. Bone. 2013;56(2):440-8. https://doi.org/10.1016/j.bone.2013.06.030.

28. Mashiba T, Hirano T, Turner $\mathrm{CH}$, et al. Suppressed bone turnover by bisphosphonates increases microdamage accumulation and reduces some biomechanical properties in dog rib. J Bone Miner Res. 2000;15(4):613-20. https://doi.org/10.1359/jbmr.2000.15.4.613

29. Wang W, Yeung KWK. Bone grafts and biomaterials substitutes for bone defect repair: A review. Bioact Mater. 2017;2(4):224 - 47. https://doi.org/10.1016/j.bioactmat.2017.05.007.

30. Solomon DH, Hochberg MC, Mogun $\mathrm{H}$, et al. The relation between bisphosphonate use and non-union of fractures of the humerus in older adults. Osteoporos Int. 2009;20(6):895-901. https://doi.org/10.1007/s00198-008-0759-z.

31. den Boer FC, Bramer JA, Blokhuis TJ, et al. Effect of recombinant human osteogenic protein-1 on the healing of a freshly closed diaphyseal fracture. Bone. 2002;31(1):158-64. https://doi.org/10.1016/s8756-3282(02)00816-5.

32. Cook SD, Baffes GC, Wolfe MW, et al. Recombinant human bone morphogenetic protein-7 induces healing in a canine long-bone segmental defect model. Clin Orthop Relat Res. 1994;(301):302-12.

33. Li F, Yu F, Liao X, et al. Efficacy of Recombinant Human BMP2 and PDGF-BB in Orofacial Bone Regeneration: A Systematic Review and Meta-analysis. Sci Rep. 2019;9(1):8073. https://doi.org/10.1038/s41598-019-44368-z.

34. Barba-Recreo P, Del Castillo Pardo de Vera JL, et al. Zoledronic acid - related osteonecrosis of the jaws. Experimental model with dental extractions in rats. Br J Oral Maxillofac Surg. 2017;55(2):1569. https://doi.org/10.1016/j.bjoms.2016.10.006.

\section{Tables}

Table 1. Radiologic union. Radiologic union was assessed by bony bridge formation of the cortex. A complete union was defined as the bony bridge formation of both cortexes in radiography. A partial union was defined as the bony bridge formation of one cortex. The numbers stated in the table (number/number) represent complete union/partial union. (one-way ANOVA, $p<0.05$. post-hoc test with Scheffe, Group I vs. Group II, $p=0.231$; Group I vs. Group III, $p<0.001$, Group I vs. Goup IV, $p<0.001$; Group II vs. Group III, $p=0.035$; Group II vs. Group IV, $p=0.044$, Group III vs. Group IV, $p=0.766$ ) 


\begin{tabular}{|lllll|}
\hline Complete/partial union & 4 wks & 8 wks & 12 wks & 16 wks \\
\hline Group I $(n=10)$ & $0 / 0$ & $0 / 1$ & $0 / 1$ & $0 / 1$ \\
\hline Group II $(n=10)$ & $0 / 2$ & $0 / 2$ & $1 / 3$ & $2 / 2$ \\
\hline Group III $(n=10)$ & $0 / 3$ & $1 / 3$ & $4 / 3$ & $6 / 3$ \\
\hline Group IV $(n=10)$ & $0 / 3$ & $1 / 4$ & $3 / 4$ & $6 / 2$ \\
\hline
\end{tabular}

Table 2. Manual test. Manual palpation with varus/valgus and anterior/posterior angulated force was performed for all harvest femora ( 16 weak after surgical procedure). When no motion was present, it was considered a complete union.

(one-way ANOVA, $p<0.05$. post-hoc test with Scheffe, Group I vs. Group II, $p=0.072$; Group I vs. Group III, $p<0.001$, Group I vs. Goup IV, $p<0.001$; Group II vs. Group III, $p=0.018$; Group II vs. Group IV, $p=0.018$, Group III vs. Group IV, $p=1.0$ )

\begin{tabular}{|lll|}
\hline Complete union & 16 wks & Union rate \\
\hline Group I $(n=10)$ & 0 & 0 \\
\hline Group II $(n=10)$ & 2 & $20 \%$ \\
\hline Group III $(n=10)$ & 6 & $60 \%$ \\
\hline Group IV $(n=10)$ & 6 & $60 \%$ \\
\hline
\end{tabular}

Table 3. Bone structure parameters - Micro CT. Bone morphometric parameters of healing of the defect sites in the femur. Data presented as mean \pm SD.

(one-way ANOVA, $p<0.05$. post-hoc test with Scheffe, Bone volume (BV) Group I vs. Group II, $p=0.304$; Group I vs. Group III, $p=0.005$, Group I vs. Goup IV, $p<0.001$; Group II vs. Group III, $p=0.026$; Group II vs. Group IV, $p<0.001$, Group III vs. Group IV, p = 0.06, Bone volume/tissue volume (BV/TV) Group I vs. Group II, $p=0.005$; Group I vs. Group III, $p<0.001$, Group I vs. Goup IV, $p=0.0034$; Group II vs. Group III, $p<$ 0.001; Group II vs. Group IV, $p=0.026$, Group III vs. Group IV, $p=0.25$ )

\begin{tabular}{|lll|}
\hline & BV $\left(\mathrm{mm}^{3}\right)$ & BV/TV $(\%)$ \\
\hline Group I $(n=10)$ & $42.8 \pm 19.8$ & $15.79 \pm 5.6$ \\
\hline Group II $(n=10)$ & $72.93 \pm 15.6$ & $36.44 \pm 2.5$ \\
\hline Group III $(n=10)$ & $168.78 \pm 20.1$ & $64.3 \pm 1.4$ \\
\hline Group IV $(n=10)$ & $193.94 \pm 61.5$ & $56.59 \pm 9.8$ \\
\hline
\end{tabular}

\section{Figures}




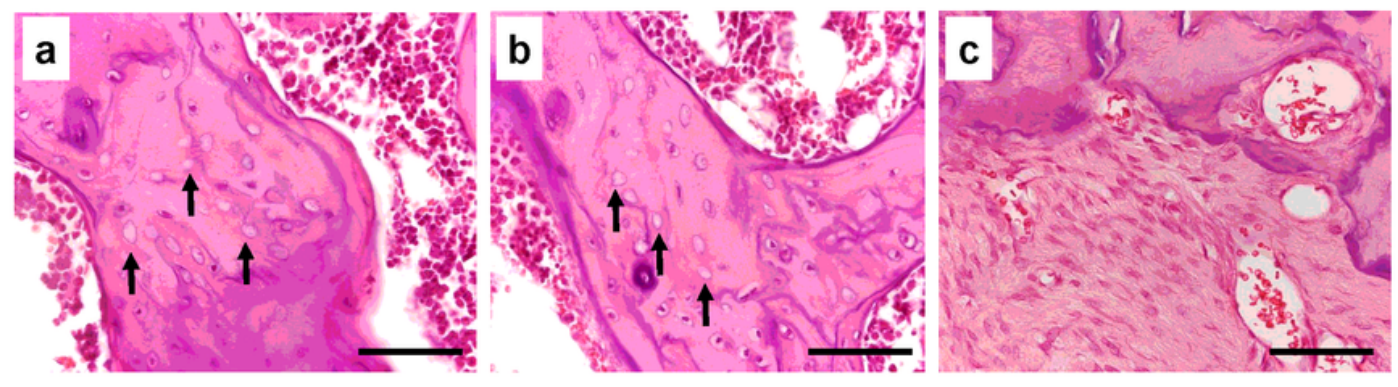

Figure 1

\section{Figure 1}

Histological analysis of distal femur. Cancellous bone shows focal osteonecrosis represented by empty lacunae (a, b: arrows) and fibrotic proliferation in the bone marrow (c). Black bars $=50 \mu \mathrm{m}$.

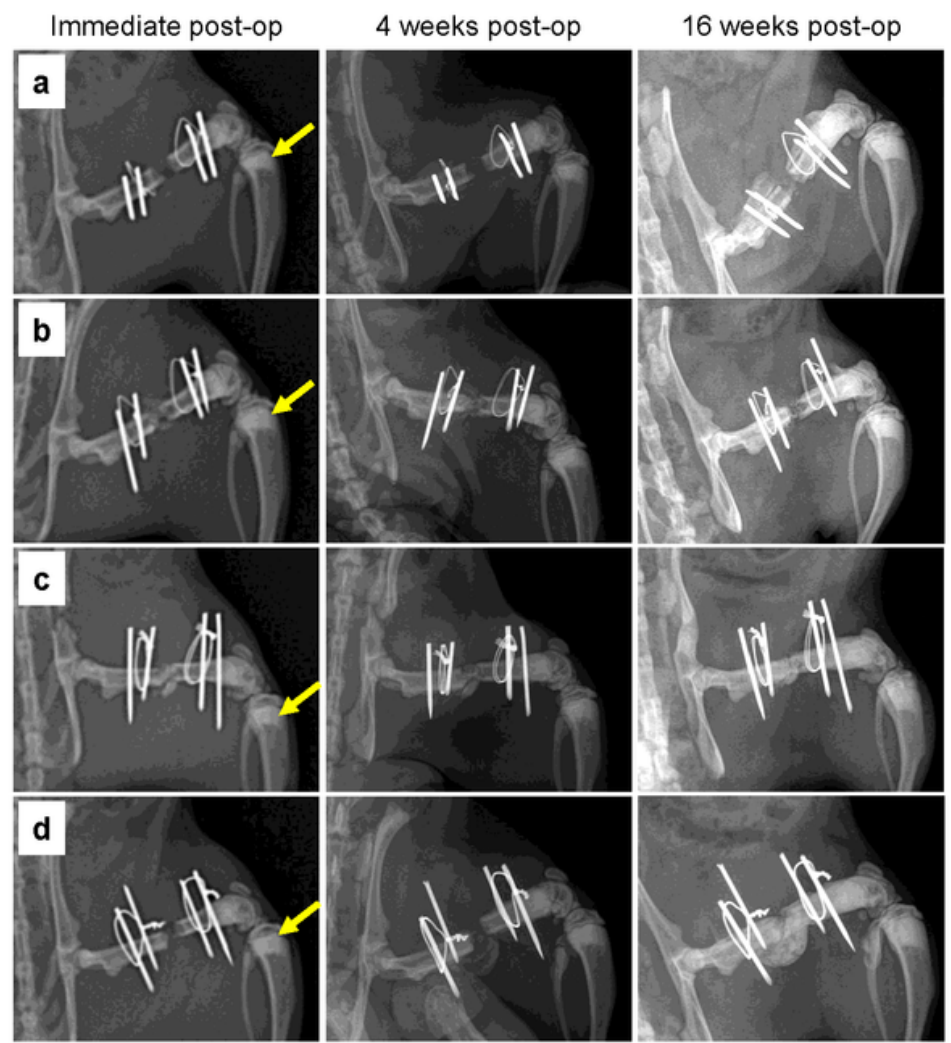


Figure 2

Radiographic findings at postoperative four and 16 weeks in each group. a- group I, b- group II, c- group III, and d-group IV. Groups I and II showed nonunion while groups III and IV showed unions in the femoral defects.

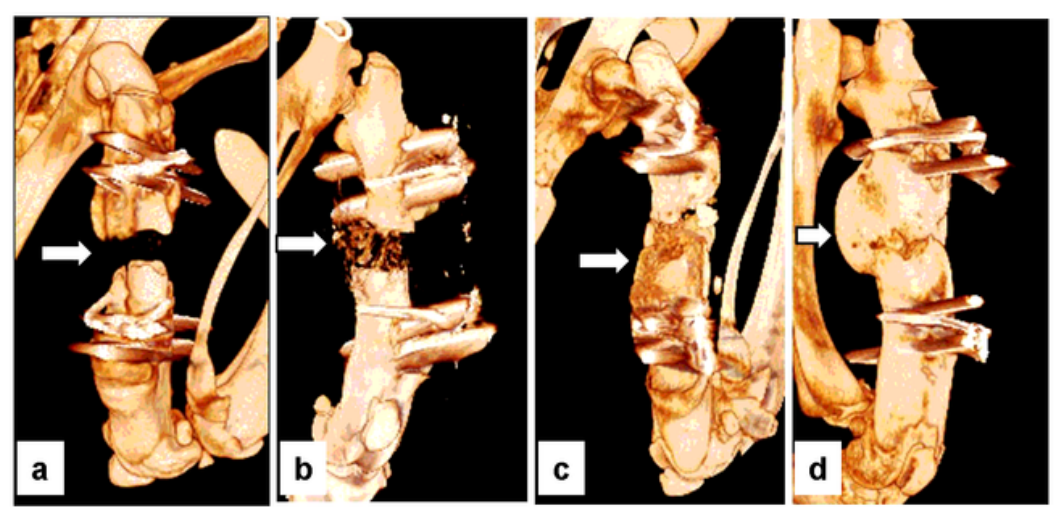

Figure 3

Figure 3

3D reconstruction images showing bone formation at the femoral defect sites in each group. A- group I, Bgroup II, C- group III, and D- group IV. No bony tissue was found In the femoral defects in group I, a small amount of bony tissue was found in group II, a large amount of bony tissue was found in group III, and the largest amount of bony tissue was found in group IV. 


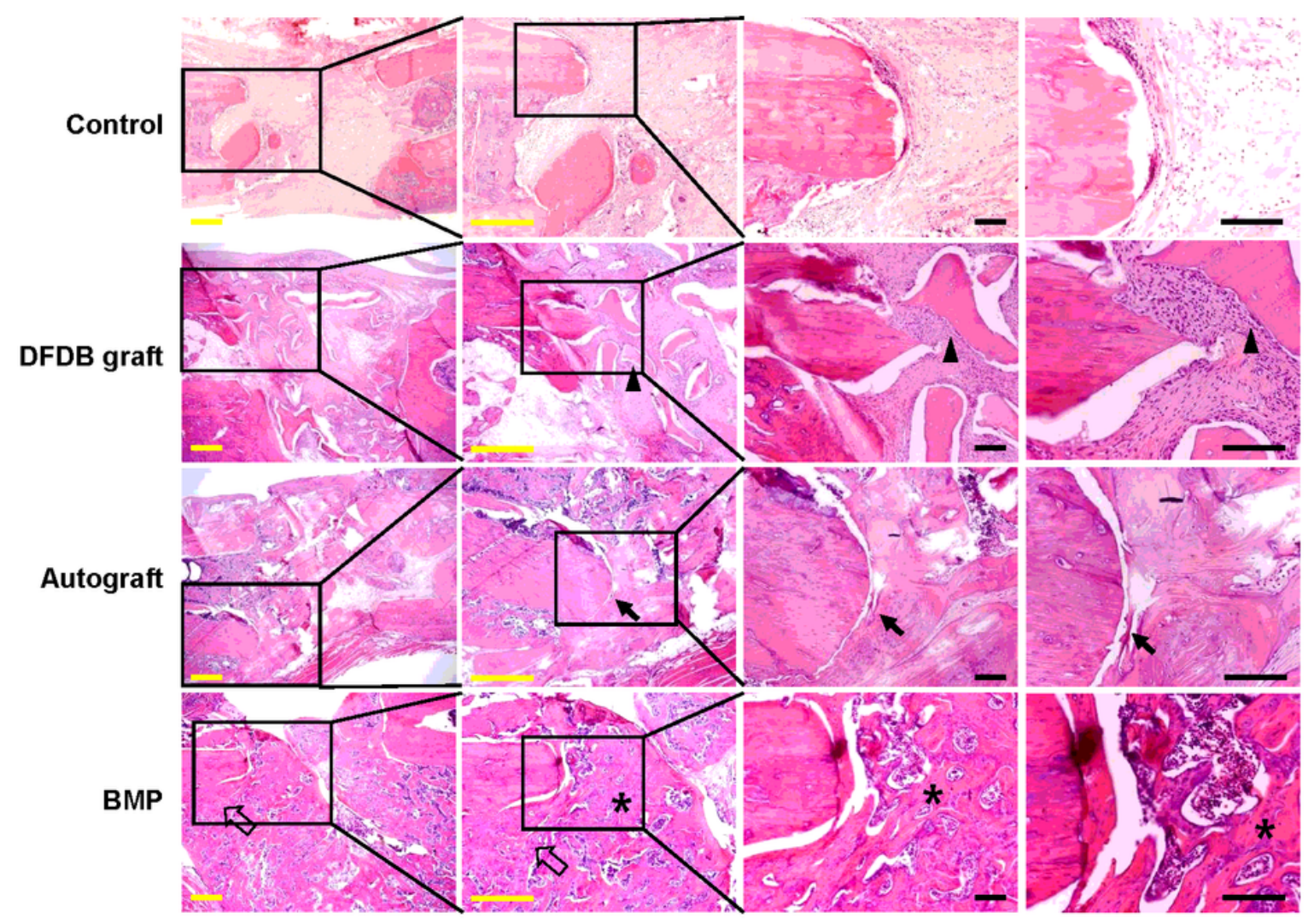

Figure 4

\section{Figure 4}

Histologic findings of the bone defect sites in the various experimental groups. The bone defect site was filled with fibrous tissue without bone formation in the absorbable collagen sponge alone group. The defect site in the demineralized freeze-dried bone (DFDB) graft group was filled with fragmented lamellar bone (the arrowhead indicates the graft bone) and fibrous tissue. The defect site in the autograft group was also filled with lamellar bone. However, the lamellar bone in the defect site with an autograft was thicker and more abundant with relatively less fibrous tissue compared to the DFDB graft group. However, the junction between the cortical bone and newly formed bone from the autograft was partially discontinuous (arrows). In contrast, the defect site in the BMP-treated group was filled with newly formed bone (asterisks) and the junction between the cortical bone and new bone was continuous (empty arrows). Yellow bars $=100 \mu \mathrm{m}$, Black bars $=50 \mu \mathrm{m}$. 